\title{
The Impact of Patient-Centered Medical Homes on Quality of Care and Medication Adherence in Patients with Diabetes Mellitus
}

\author{
JaeJin An, BPharm, PhD
}

\begin{abstract}
BACKGROUND: Current evidence suggests that patient-centered medical homes (PCMHs) potentially increase overall quality of disease management, including preventive care. However, there are mixed findings regarding quality of diabetes care, and little evidence exists with respect to the effect of PCMHs on medication adherence in patients with diabetes.

OBJECTIVE: To investigate associations between PCMHs and process measures of diabetes care, as well as adherence to oral hypoglycemic agents (OHAs) in patients with diabetes in a nationally representative U.S. sample.

METHODS: Using the 2009-2013 longitudinal data files from the Medical Expenditure Panel Survey, adult diabetes patients were identified. Patients whose usual sources of care have all PCMH features at baseline (i.e., enhanced access after hours and online, shared decision making, and enhanced patient engagement) were categorized as the PCMH group, which was compared with patients without PCMH features. Process measures of diabetes care included $\geq 2$ hemoglobin A1c tests and $\geq 1$ cholesterol test, foot examination, dilated eye examination, and flu vaccination during 1 year of follow-up. Medication possession ratio (MPR) during follow-up was calculated for patients with $\mathrm{OHAs}$ without any insulin use, with $\mathrm{MPR} \geq 80 \%$ considered to be adherent to $\mathrm{OHAs}$. Univariate and multivariate regression models considering sampling strata and weights were used to examine the association between the PCMH and study outcomes.
\end{abstract}

RESULTS: A total of 3,334 patients with diabetes was identified, representing 61 million U.S. lives. The mean (SE) age was $61.6(0.3)$ years, and $52.4 \%$ of patients were female. The mean (SE) years of having diabetes was 12.0 (0.2) years. Approximately $11.4 \%$ of the patients were categorized as the PCMH group at baseline, and only $3.6 \%$ of those patients remained in the PCMH group for 2 years. Of the diabetic patients identified, only $26.9 \%$ met all of the diabetes care process measure criteria defined in this study. A higher proportion of patients met process measure criteria in the PCMH group compared with the non-PCMH group $(33.8 \%$ vs. $26.0 \%$, respectively, $P=0.015)$. The weighted mean MPR $(95 \% \mathrm{Cl})$ of $\mathrm{OHAs}$ from the 2 groups were not statistically different $(0.68,95 \% \mathrm{Cl}=0.63-0.74$ for the PCMH group; $0.77,95 \% \mathrm{Cl}=0.72-0.82$ for the non-PCMH group, $P=0.675$ ), resulting in $47.4 \%$ of adherent patients in the study population. Overall, the PCMH group was associated with improvement in the process measures of diabetes care (adjusted odds ratios $[\mathrm{OR}]=1.42,95 \% \mathrm{Cl}=1.06-1.91$ ). The effect was greater among the patients who stayed in the PCMH group for 2 years $(O R=2.09,95 \% \mathrm{Cl}=1.27-3.45)$; were aged $<65$ years $(O R=2.19$, $95 \% \mathrm{Cl}=1.47-3.28)$; or had less than 3 comorbid conditions $(\mathrm{OR}=2.38$, $95 \% \mathrm{Cl}=1.46-3.88)$. However, the PCMH group was not associated with an increased adherence to OHAs $(P=0.495)$ or an increase in MPR $(P=0.570)$.

CONCLUSIONS: Patients with all PCMH features were associated with improvement in the process measures of diabetes care, but not in adherence to OHAs. Future studies should investigate the mechanism of PCMHs in overall quality of care, as well as medication adherence.

J Manag Care Spec Pharm. 2016;22(11):1272-84

Copyright $\odot 2016$, Academy of Managed Care Pharmacy. All rights reserved.

\section{What is already known about this subject}

Recent evaluation studies of patient-centered medical homes (PCMHs) suggest positive effects on preventive care services, but there are mixed findings regarding quality of care, with limited evidence of medication adherence.

The PCMH model provides a potential mechanism for improving medication adherence through enhanced coordination, case management, medication reconciliation, as well as shared decision making, and enhanced access to care.

\section{What this study adds}

The PCMH care model was associated with improvement in process measures of diabetes care mainly because of increased rate of dilated eye examinations. Enhanced shared decision making and patient engagement features of the PCMH model likely influenced these findings.

The effect of PCMHs seems to be greater in the younger population, as well as in patients with less comorbid conditions.

The PCMH study group was not associated with an increased adherence to oral hypoglycemic agents, which suggests diverse effects of the PCMH model on different outcomes.

$\mathrm{P}$ atient-centered medical homes (PCMHs), or medical homes, have been suggested as a novel way to improve quality of care, patient and caregiver experience, and affordability. ${ }^{1}$ The term "medical home" first appeared in Standards of Child Health Care, which was published by the American Academy of Pediatrics in 1967 and emphasized the importance of centralized medical records for children with special health care needs. ${ }^{2}$ The current concept of PCMHs has been greatly expanded and is based on previous efforts to redesign primary care to provide for better quality of care. ${ }^{3}$ The PCMH model puts more emphasis on primary care physicians, who are responsible for leading interdisciplinary teams and encouraging cooperation and collaboration between providers and patients. ${ }^{4}$ Along with other organizations, the National Committee for Quality Assurance (NCQA) defined the joint principles of the PCMH model, which include a personal physician, physician-directed medical practice, coordinated and/or integrated care, whole-person orientation, high quality and safe care, enhanced access, and appropriate reimbursement for PCMH services. ${ }^{4}$ The PCMH model has received growing 
attention-nearly 38,000 clinicians and 7,500 sites or clinics were recognized by the NCQA, and another 1,286 sites were certified by The Joint Commission as of June 2014. ${ }^{5}$

The literature examining the effects of PCMH interventions on utilization, quality of care, and health care costs is growing. Systematic reviews and recent studies have reported positive effects of the PCMH model on preventive care services such as cancer screening and vaccinations. ${ }^{1,6}$ However, studies show mixed findings regarding quality of care. ${ }^{6-13}$ Friedberg et al. (2015) found higher rates of process measures of diabetes care in the PCMH group compared with the usual care group, ${ }^{12}$ while Rosenthal et al. (2015) reported no differences in Healthcare Effectiveness Data and Information Set (HEDIS) quality measures of diabetes care between the PCMH care and usual care groups. ${ }^{10}$ Moreover, little evidence exists that shows reduction of emergency department visits and overall cost savings from PCMH interventions. ${ }^{1,6,10,12,14-16}$

The PCMH model also provides a potential mechanism for improving medication adherence through enhanced coordination, case management, and medication reconciliation, ${ }^{17}$ as well as shared decision making and access to care. Several studies have investigated the association between the PCMH model and increased use of medication therapy. Domino et al. (2009) showed that PCMH care for asthma improved the use of maintenance and rescue medications. ${ }^{18}$ Beadles et al. (2014) found a positive association between PCMHs and initiation of oral anticoagulation therapy. ${ }^{19}$ Beadles et al. (2015) reported a small absolute increase in proportion of days covered (PDC; a 4.8 percentage point increase $)^{17}$ for PCMH enrollees, compared with non-PCMH enrollees, with multiple chronic conditions. More evidence is needed to further understand the effect of the PCMH care model on medication adherence.

This study investigated the effect of the PCMH model on patients with diabetes using longitudinal Medical Expenditure Panel Survey (MEPS) data files. The PCMH model is particularly applicable to diabetes care, since most patients with diabetes are managed by primary care providers, and many PCMH pilot programs focus on diabetes, such as the Chronic Care Initiative. ${ }^{12,20}$ MEPS collects data from a national U.S. representative sample, and the data source has the benefit of understanding patient perception of PCMHs. ${ }^{21}$

The purpose of this study was to assess the association between PCMHs and process measures of diabetes care, as well as adherence to oral hypoglycemic agents (OHAs) in patients with diabetes. The study hypothesized that patients whose usual sources of care have all PCMH features would have greater diabetes screening and/or exam rates and prescription medication use; therefore, the PCMH care model would improve the performance of diabetes process measures and increase medication adherence.

\section{Methods}

\section{Data Source and Study Sample}

MEPS is a nationally representative survey of the civilian noninstitutionalized population of all ages in the United States. Conducted by the Agency for Healthcare Research and Quality, MEPS collects comprehensive data on health care utilization and expenditures. Data are collected from over 5 rounds of in-person interviews that cover a consecutive 2-year period. Patients with diabetes mellitus were identified using Clinical Classification Codes 049 and 050 (diabetes mellitus with or without complications) in medical condition files from the years 2009-2012. Among these patients, only respondents in the MEPS longitudinal files (MEPS panels 14, 15, 16, and 17) were included. Each longitudinal file has 2 years of data (year 1 and year 2). This study considered year 1 survey results as the baseline period and year 2 survey results as the follow-up period. PCMH features were determined at baseline (year 1), and study outcomes were assessed during follow-up (year 2). Age exclusion criteria (aged $<18$ years at baseline) were further applied so only adult diabetic patients were included in the study. The final study sample included individuals who had usual care providers for both years and who responded "yes" for diabetes diagnosis by health professionals at baseline. For the medication adherence outcome, the analysis was restricted to individuals with at least 1 OHA (therapeutic subclass $=99)^{22}$ at baseline without any insulin use during follow-up (therapeutic subclass $=215$ or "no" for the question of whether they were treated with insulin injections). Patients who were on insulin therapy at baseline but no longer received insulin during follow-up ( $2.7 \%$ of the population) were included, since adherence to OHA calculation was not affected by baseline insulin use.

\section{Definition of PCMH}

The PCMH care model was defined using the provider-related questionnaires in MEPS. These questionnaires included questions regarding enhanced access to the usual care provider after hours and online, shared decision making, and enhanced patient engagement. The following selected questions were adopted from previous literature that investigated the effectiveness of PCMH in MEPS21,23-25:

1. "How difficult is it to contact [medical provider] after regular hours in case of urgent medical needs?"

2. "Does [medical provider] have office hours at night or on weekends?"

3. "How difficult is it to contact [medical provider] during regular business hours over the telephone about a health problem?" 
4. "How often would [medical provider] ask patient to help make the decision?"

5. "Does [medical provider] usually ask about prescription medications and treatments other doctors may give them?"

The responses were categorized as (a) a positive response ("usually/always" for frequency questions and "not too difficult/ not at all difficult" for difficulty questions); (b) missing ("don't know/refused"); and (c) a negative response for others. Patients with positive responses to all 5 questions were categorized as the PCMH group; all others were considered the non-PCMH group.

\section{Outcomes}

Process Measures of Diabetes Care. The American Diabetes Association (ADA) recommends the following for diabetes care: (a) 2 or more hemoglobin Alc laboratory tests, (b) cholesterol tests, (c) a foot examination, (d) a dilated eye examination, and (e) flu vaccination every year. ${ }^{26}$ Consistent with ADA recommendations, responses to these 5 process measures of diabetes care were used to define optimal diabetes care. Patients who were adherent to all $5 \mathrm{ADA}$ recommendations were considered optimal. The selected questions from the diabetes care survey in the MEPS household component are as follows:

1. "During [year], how many times did a doctor, nurse, or other health professional check your blood for glycosylated hemoglobin or "hemoglobin Alc'?"

2. "Which of the following year(s) did you have your blood cholesterol checked?"

3. "Which of the following year(s) did a doctor or other health professional check your feet for any sores or irritations?"

4. "Which of the following year(s) did you have an eye exam in which your pupils were dilated?"

5. "Which of the following year(s) did you get a flu vaccination?"

Medication Adherence. Medication possession ratio (MPR) during 1 year of follow-up was calculated for patients with at least 1 OHA at baseline without any insulin use. The MPR was calculated using prescription pharmacy records in MEPS. Baseline OHAs were categorized using therapeutic subclasses that included biguanides, sulfonylureas, alpha glucosidase inhibitors, thiazolidinediones, meglitinides, combination agents, and dipeptidyl peptidase-4 inhibitors. Assuming that patients were expected to continue all baseline subclasses of OHAs during follow-up, the average MPR for each OHA class was calculated. The MPR was calculated by summing the days supply for each OHA class and dividing by 365 days. Then, the average MPR was calculated using MPRs for each OHA class. The calculated MPR was truncated at $100 \%$, and MPR $\geq 80 \%$ was considered adherent to any OHA. ${ }^{27}$ For example, if a patient had metformin (biguanides) and glimepiride (sulfonylurea) prescriptions at baseline, the MPR of metformin and the MPR of glimepiride were calculated separately and then the average of both MPRs was calculated. This method allowed for switch therapy between drugs within the same class. If the patient switched from glimepiride to glipizide (sulfonylurea), the days supply of both drugs (glimepiride or glipizide) were included in calculating the MPR of sulfonylurea. For patients who switched to different classes of OHAs during follow-up without any baseline OHA use, only MPRs of the new drug classes were calculated. For example, if a patient had metformin (biguanides) prescriptions, but only had rosiglitazone (thiazolidinedione) prescriptions during follow-up, the MPR of rosiglitazone was calculated, assuming that this patient had switched from metformin to rosiglitazone. For patients who added another class of OHA, only the average MPR of baseline OHAs was calculated, since this augmentation might happen in the middle of follow-up.

Approximately $31.5 \%$ of the pharmacy records had missing days supply or incorrect data (days supply of zero). Four methods were used to impute missing values: (1) the same days supply when the same drug name and the same quantity for the same individual was available $(5.0 \%$ of the total missing values); (2) the calculated days supply when the same drug name for the same individual was available, applying the same dose per day (5.2\% of the missing values); (3) the calculated days supply applying the most frequent dose per day per OHA from the data ( $83.6 \%$ of the missing values); and (4) the most frequent days supply, applying the most frequent number of pills per day per OHA and quantity from the data $(6.3 \%$ of missing values). This missing imputation strategy was tested in the sensitivity analyses.

\section{Covariates}

Covariates included in the model were selected a priori based on adherence literature and availability of data. Patient sociodemographic variables were included, such as age, race/ ethnicity, sex, marital status, education, and region. Perceived health of patients, as well as years of diabetes and insulin dependence (only for the diabetes process measure analysis) were considered as covariates. Disease severity was controlled using a Elixhauser comorbidity score or number of comorbid conditions to consider multiple chronic conditions. ${ }^{28-30}$ For the adherence outcome, a few additional covariates were considered, such as number of OHA classes (proxy for polypharmacy), use of $\geq 1$ brand-name OHA (defined by using National Drug Code numbers), copayment for OHAs, and depression at baseline. The list of covariates was finalized after a multicollinearity check using the variance inflation factor.

\section{Statistical Analyses}

Descriptive statistics (t-tests for continuous variables and RaoScott chi-square tests for categorical variables) were conducted to compare the PCMH and non-PCMH groups. Univariate and multivariate logistic regression models considering sampling strata and weights were used to examine the association 


\section{TABLE 1 Baseline Characteristics}

\begin{tabular}{|c|c|c|c|c|c|c|c|}
\hline & \multicolumn{2}{|c|}{ PCMH No } & \multicolumn{2}{|c|}{ PCMH Yes } & \multicolumn{2}{|c|}{ Total } & \multirow[b]{2}{*}{$P$ Value } \\
\hline & $\mathrm{N}$ & Weighted \% & $\mathrm{N}$ & Weighted \% & $\mathrm{N}$ & Weighted \% & \\
\hline Overall Cohort & \multicolumn{2}{|c|}{$\begin{array}{c}(\mathrm{n}=2,956 ; \text { weighted } \\
\mathrm{n}=54 \text { million })\end{array}$} & \multicolumn{2}{|c|}{$\begin{array}{c}(\mathrm{n}=378 ; \text { weighted } \\
\mathrm{n}=6.9 \text { million })\end{array}$} & \multicolumn{2}{|c|}{$\begin{array}{l}(\mathrm{N}=3,334 ; \text { weighted } \\
\mathrm{N}=61 \text { million })\end{array}$} & \\
\hline \multicolumn{8}{|l|}{ Panel } \\
\hline 14 & 718 & 23.3 & 71 & 19.8 & 789 & 22.9 & 0.5577 \\
\hline 15 & 649 & 27.2 & 88 & 30.3 & 737 & 27.6 & \\
\hline 16 & 826 & 24.6 & 114 & 25.3 & 940 & 24.7 & \\
\hline 17 & 763 & 24.9 & 105 & 24.6 & 868 & 24.9 & \\
\hline Mean age (SE) & & $61.7(0.3)$ & & $61.5(0.9)$ & & $61.6(0.3)$ & 0.8894 \\
\hline $18-45$ years & 356 & 11.0 & 43 & 11.6 & 399 & 11.0 & 0.9208 \\
\hline 45-64 years & 1,368 & 45.0 & 174 & 45.6 & 1,542 & 45.1 & \\
\hline$\geq 65$ years & 1,232 & 44.0 & 161 & 42.8 & 1,393 & 43.9 & \\
\hline \multicolumn{8}{|l|}{ Sex } \\
\hline Male & 1,305 & 47.9 & 169 & 45.1 & 1,474 & 47.6 & 0.3457 \\
\hline Female & 1,651 & 52.1 & 209 & 54.9 & 1,860 & 52.4 & \\
\hline \multicolumn{8}{|l|}{ Race/ethnicity } \\
\hline White & 1,264 & 64.0 & 155 & 65.4 & 1,419 & 64.2 & 0.9493 \\
\hline Black & 765 & 15.4 & 88 & 14.7 & 853 & 15.3 & \\
\hline Nonwhite Hispanic & 672 & 13.5 & 95 & 13.6 & 767 & 13.6 & \\
\hline Asian/others & 255 & 7.0 & 40 & 6.3 & 295 & 7.0 & \\
\hline \multicolumn{8}{|l|}{ Spouse/marital status } \\
\hline Spouse in the house & 1,534 & 56.6 & 200 & 56.8 & 1,734 & 56.7 & 0.9642 \\
\hline Not married/no spouse & 1,422 & 43.4 & 178 & 43.2 & 1,600 & 43.3 & \\
\hline \multicolumn{8}{|l|}{ Education } \\
\hline Lower than high school & 1,719 & 55.6 & 205 & 54.5 & 1,924 & 55.5 & 0.8667 \\
\hline High school & 465 & 19.2 & 67 & 20.8 & 532 & 19.4 & \\
\hline College or more & 772 & 25.2 & 106 & 24.7 & 878 & 25.1 & \\
\hline \multicolumn{8}{|l|}{ Region } \\
\hline Northeast & 456 & 17.1 & 78 & 21.2 & 534 & 17.5 & 0.0346 \\
\hline Midwest & 575 & 22.3 & 77 & 27.5 & 652 & 22.9 & \\
\hline South & 1,274 & 41.6 & 113 & 30.2 & 1,387 & 40.3 & \\
\hline West & 651 & 19.0 & 110 & 21.1 & 761 & 19.3 & \\
\hline \multicolumn{8}{|l|}{ Perceived health status } \\
\hline Excellent/good & 238 & 9.3 & 30 & 9.5 & 268 & 9.3 & 0.9100 \\
\hline Good & 947 & 35.4 & 127 & 36.7 & 1,074 & 35.5 & \\
\hline Fair/poor & 1,771 & 55.3 & 221 & 53.7 & 1,992 & 55.1 & \\
\hline Years of diabetes & & $11.9(0.3)$ & & $12.8(0.9)$ & & $12.0(0.3)$ & 0.3785 \\
\hline \multicolumn{8}{|l|}{ Insulin use } \\
\hline Yes & 902 & 29.9 & 140 & 36.4 & 1,042 & 30.7 & 0.0745 \\
\hline No & 2,036 & 69.6 & 234 & 62.7 & 2,270 & 68.8 & \\
\hline Not available & 18 & 0.5 & 4 & 0.9 & 22 & 0.5 & \\
\hline Number of chronic conditions, mean (SE) & & $3.6(0.04)$ & & $3.5(0.1)$ & & $3.5(0.03)$ & 0.5667 \\
\hline 1 & 249 & 7.9 & 49 & 12.5 & 298 & 8.4 & 0.9343 \\
\hline 2 & 528 & 17.2 & 68 & 16.5 & 596 & 17.1 & \\
\hline 3 & 845 & 27.0 & 100 & 22.2 & 945 & 26.4 & \\
\hline 4 & 640 & 22.8 & 80 & 24.5 & 720 & 23.0 & \\
\hline 5 or more & 694 & 25.1 & 81 & 24.3 & 775 & 25.0 & \\
\hline Mean Elixhauser comorbidity score (SE) & & $3.2(0.14)$ & & $3.2(0.39)$ & & $3.2(0.13)$ & 0.9061 \\
\hline \multicolumn{8}{|l|}{ Comorbidity } \\
\hline Hypertension & 2,254 & 74.3 & 281 & 74.0 & 2,535 & 74.3 & 0.9343 \\
\hline Dyslipidemia & 1,981 & 67.7 & 238 & 65.0 & 2,219 & 67.4 & 0.4203 \\
\hline Coronary artery disease & 590 & 20.7 & 73 & 22.0 & 663 & 20.9 & 0.6274 \\
\hline Arthritis & 581 & 20.3 & 69 & 21.1 & 650 & 20.4 & 0.7944 \\
\hline Depression & 446 & 16.6 & 51 & 15.2 & 497 & 16.4 & 0.5745 \\
\hline Cancer & 301 & 12.1 & 50 & 15.1 & 351 & 12.4 & 0.1645 \\
\hline
\end{tabular}


TABLE 1 Baseline Characteristics (continued)

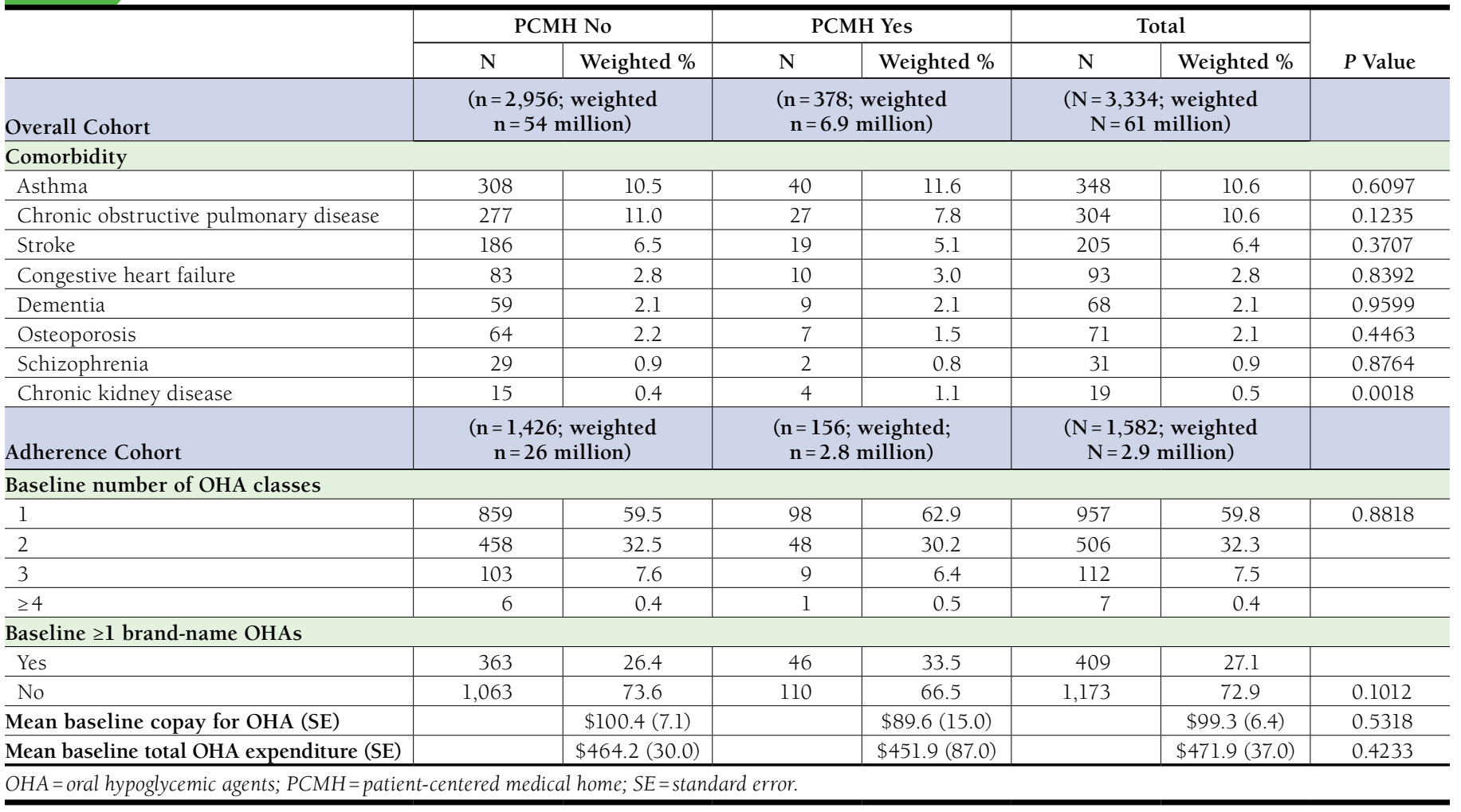

between PCMHs and diabetes process measures, as well as medication adherence. Potential confounding factors previously listed were tested and added to the multivariate logistic regression model as covariates.

A priori subgroup analyses were conducted to test whether the effect of PCMHs is consistent across the different subgroups of the population. Age group (aged over 65 years, less than 65 years) and number of comorbid conditions (3 or more, less than 3) were considered in this analysis. Sensitivity analyses were conducted using different MPR cutoffs for the definition of medication adherence, MPR of any OHA, excluding missing values in days supply, and MPR as a continuous variable. A $P$ value of $<0.05$ was considered statistically significant. All statistical analyses were performed using SAS, version 9.4 (SAS Institute, Cary, NC).

\section{Results}

A total of 3,334 patients with diabetes was identified, representing 61 million U.S. lives, by applying survey weights. The mean (standard error [SE]) age was $61.6(0.3)$ years, and $52.4 \%$ of the patients were female. The mean (SE) duration of diabetes was $12.0(0.3)$ years; $30.7 \%$ of the patients were on insulin at baseline, and the mean (SE) number of comorbid conditions was $3.5(0.03)$ (Table 1). A higher proportion of patients in the PCMH group were in the Northeast/Midwest regions. Approximately, $11.4 \%$ of the patients were categorized as a PCMH group at baseline, and $88.6 \%$ were considered as the non-PCMH group. For the adherence outcome evaluation, 1,582 patients were identified after applying additional exclusion criteria, and $9.8 \%$ of that number were considered as PCMH patients, with $90.2 \%$ considered as non-PCMH patients.

The majority of patients (about $80 \%$ ) responded that their usual care providers ask patients to help decide between treatments, ask about treatment prescribed by other doctors, and they have no or less difficulty in contacting their providers by phone. However, a relatively smaller percentage of patients (28.2\%-43.8\%) responded that they have no difficulty in contacting their providers after hours, at night, or on weekends. Only $3.6 \%$ of the patients remained in the PCMH group over the 2 years. During the follow-up year, a large proportion of patients changed their responses to "no" or "unknown" because they experienced difficulty contacting physicians after hours or on nights/weekends during follow-up (Table 2).

Table 3 summarizes the process measures of diabetes care and adherence to OHAs by the PCMH group. Although $50 \%-80 \%$ of each process measure criteria were met, only $26.9 \%$ of the diabetic patients met all diabetes process measure performance criteria defined in this study. Alc testing had the lowest 
TABLE 2 Summary of Patient-Centered Medical Home Status

\begin{tabular}{|c|c|c|c|c|c|c|}
\hline & \multicolumn{2}{|c|}{ Positive } & \multicolumn{2}{|c|}{ Negative } & \multicolumn{2}{|c|}{ Unknown } \\
\hline & $\mathrm{N}$ & Weighted \% & $\mathrm{N}$ & Weighted \% & $\mathrm{N}$ & Weighted \% \\
\hline Response at Baseline (Year 1) & \multicolumn{6}{|c|}{ Response at Baseline (Year 1) } \\
\hline Difficulty contacting after hours & 1,458 & 43.8 & 927 & 25.2 & 949 & 31.0 \\
\hline Difficulty contacting nights/weekends & 930 & 28.2 & 2,111 & 63.3 & 293 & 8.5 \\
\hline Difficulty contacting over the phone & 2,690 & 81.2 & 547 & 16.2 & 97 & 2.6 \\
\hline Ask patient to help decide between treatments & 2,603 & 78.9 & 521 & 14.6 & 210 & 6.5 \\
\hline Ask about treatments prescribed by other doctors & 2,691 & 80.0 & 563 & 17.6 & 80 & 2.4 \\
\hline Positive Response at Baseline (Year 1) & \multicolumn{6}{|c|}{ Response in Follow-up (Year 2) } \\
\hline Difficulty contacting after hours & 866 & 59.9 & 307 & 19.3 & 285 & 20.8 \\
\hline Difficulty contacting nights/weekends & 557 & 62.3 & 311 & 31.4 & 62 & 6.2 \\
\hline Difficulty contacting over the phone & 2,308 & 86.0 & 319 & 11.5 & 63 & 2.5 \\
\hline Ask patient to help decide between treatments & 2,167 & 84.5 & 348 & 12.1 & 88 & 3.4 \\
\hline Ask about treatments prescribed by other doctors & 2,263 & 85.1 & 381 & 13.2 & 47 & 1.7 \\
\hline
\end{tabular}

percentage (50.5\%), and cholesterol testing had the highest percentage (86.3\%). A higher proportion of patients met process measure criteria in the PCMH group compared with the nonPCMH group (33.8\% vs. $26.0 \%$, respectively, $P=0.015)$. Higher rates of dilated eye examinations were observed in the $\mathrm{PCMH}$ group, compared with the non-PCMH group (73.3\% vs. 64.7\%, respectively, $P=0.015$ ); however, other exam or testing rates were not statistically significantly different in both groups.

The weighted mean MPR (95\% confidence interval [CI]) for the OHAs were not statistically different in both groups. The mean MPR (95\% CI) of baseline OHAs was 0.68 (0.63-0.74) for the PCMH group and 0.77 (0.72-0.82) for the non-PCMH group $(P=0.675)$. The proportion of patients adherent to OHAs was relatively low and not statistically different between the PCMH and non-PCMH groups (44.9\% vs. $47.6 \%$, respectively, $P=0.565)$. The proportion was slightly higher when calculating adherence to any OHA (60.9\% vs. $63.4 \%$, respectively, $P=0.557)$.

Univariate and multivariate analyses showed that the PCMH group was associated with improvement in overall process measures of diabetes care (adjusted odds ratio $[\mathrm{OR}]=1.42,95 \%$ $\mathrm{CI}=1.06-1.91$; Figure 1 and Appendix A, available in online article). The PCMH group was associated with improvement in dilated eye exams (OR $=1.49,95 \% \mathrm{CI}=1.08-2.05)$. All the other process measures were not statistically significant. White race, higher level of education, perceived good health condition, longer duration of diabetes, and higher Elixhauser comorbidity score were also associated with better performance of the diabetes process measures (Appendix A). The effect was greater in patients who stayed in the PCMH group for 2 years (OR $=2.09$, 95\% CI=1.29-3.45; Figure 1). Investigation of each specific PCMH principle showed that asking patients to help decide between treatments $(\mathrm{OR}=1.34,95 \% \mathrm{CI}=1.11-1.60)$ and asking patients about their treatments from other providers $(\mathrm{OR}=1.45$, 95\% CI $=1.14-1.85)$ were highly associated with diabetes process measures. Subgroup analysis further reported that the effect of PCMH principles was greater in patients with less comorbid conditions (less than 3; OR $=2.38,95 \% \mathrm{CI}=1.46$ 3.88) and in the younger patient population (aged $<65$ years; $\mathrm{OR}=2.19,95 \% \mathrm{CI}=1.47-3.28$ ).

However, the PCMH group was not associated with an increased adherence to OHAs (OR=0.87, 95\% CI=0.58-1.30; Figure 2 and Appendix B, available in online article) or an increase in MPR $(P=0.570)$. Older age and higher OHA copayment were associated with medication adherence (Appendix B). Subgroup analyses and sensitivity analyses consistently showed no statistically significant association between PCMH care and medication adherence.

\section{Discussion}

This study suggests that PCMHs may improve the performance of diabetes process measures. The PCMH group was associated with a $42 \%$ higher likelihood of compliance with the ADArecommended diabetes process measures. These findings are mostly driven by the improvement of dilated eye exam rates rather than Alc or cholesterol tests. Foot exams and flu vaccinations showed positive increases; however, these findings were not statistically significant. In addition, the study findings also indicated a potential long-term effect of PCMH principles on the process measure outcome. Patients in the PCMH group for 2 consecutive years showed a higher likelihood of better performance on the diabetes care process measures. Although there are mixed results in diabetes quality of care, $, 10,15$ the findings in this study strengthen some of the previous study conclusions. A meta-analysis of earlier PCMH evaluation studies showed small-to-moderate positive effects on the delivery of preventive care services. ${ }^{6}$ More recent PCMH pilot programs reported improvement in quality measures of diabetes, and the effect seemed to be greater in long-term follow-up. . $^{12,13}$ Differences between the PCMH pilot and comparison practices became larger after 3 years of follow-up in most of the quality 
TABLE 3 Process Measures of Diabetes Care and Medication Adherence by the PCMH Group

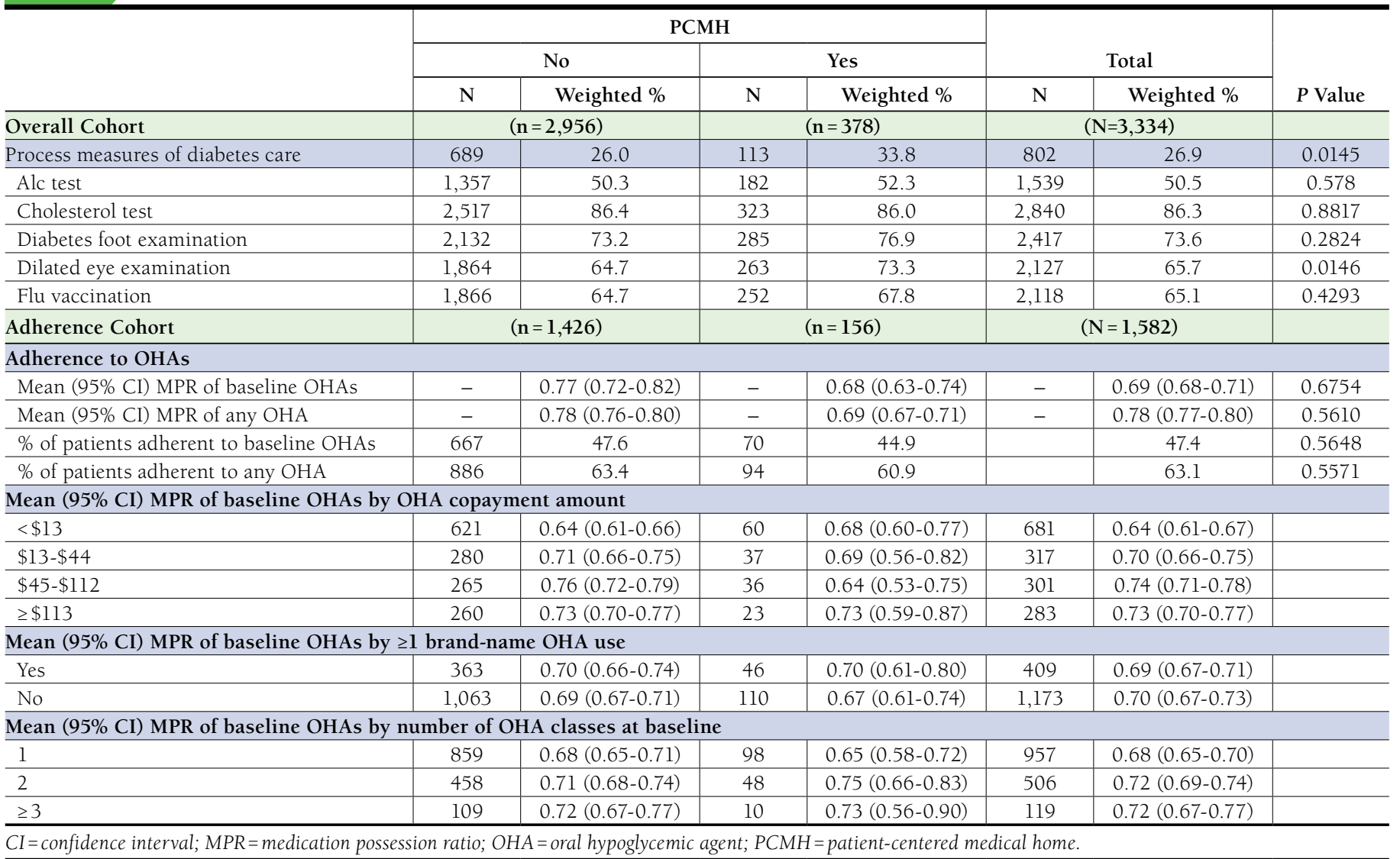

measures. ${ }^{12,13}$ These findings are consistent across diabetes process measures such as Alc testing, cholesterol testing, nephropathy monitoring, and eye examinations.

This study did not find that the PCMH model improved adherence to OHAs. This finding was consistent across subgroup analyses and sensitivity analyses. One reason for this lack of effectiveness may be because this study evaluated medication adherence among the prevalent diabetes population. The mean duration of diabetes in this population was 12 years, and this study identified patients who already had initiated OHAs at baseline. The medication-taking behavior of these patients may have already formed; therefore, the PCMH model might be less likely to influence medication adherence. The current evidence for the effect of the PCMH model on medication adherence among prevalent patients is limited. The 2015 Beadles et al. study found a small increase in PDC of newly initiating diabetes medications in patients with multiple chronic conditions, ${ }^{17}$ and the 2014 Beadles et al. study also found an association between the PCMH model and a higher rate of initiating oral anticoagulation therapy in new patients with atrial fibrillation. ${ }^{19}$
These mixed study findings suggest the necessity of understanding the effect of the PCMH model on various outcome measures. The PCMH care model focuses on coordinated and/or integrated care, whole-person orientation, and enhanced access to care. These features may be effective in preventive care or in process measures that require coordination with other health care professionals. Dilated eye examinations by ophthalmologists or optometrists is a good example of coordinated care; enhanced access and shared decision making might increase dilated eye exam rates. On the other hand, patients with diabetes who already have initiated their medications may not be influenced as much because they had better access to primary care and/or better chances of shared decision making. Instead, the PCMH principles may be more important in newly initiating therapy or transitioning of care. Other features of the PCMH model, such as adoption of electronic health records or use of case managers, might have more of an effect on improving medication adherence. Futures studies will be helpful to further understand the effect of the PCMH care model on different outcomes so that, ultimately, useful interventions can be designed for each outcome. 


\section{FIGURE 1 Adjusted Odds Ratios (95\% Cl) for Each PCMH Feature and Subgroup Analyses}

on Process Measures of Diabetes Care

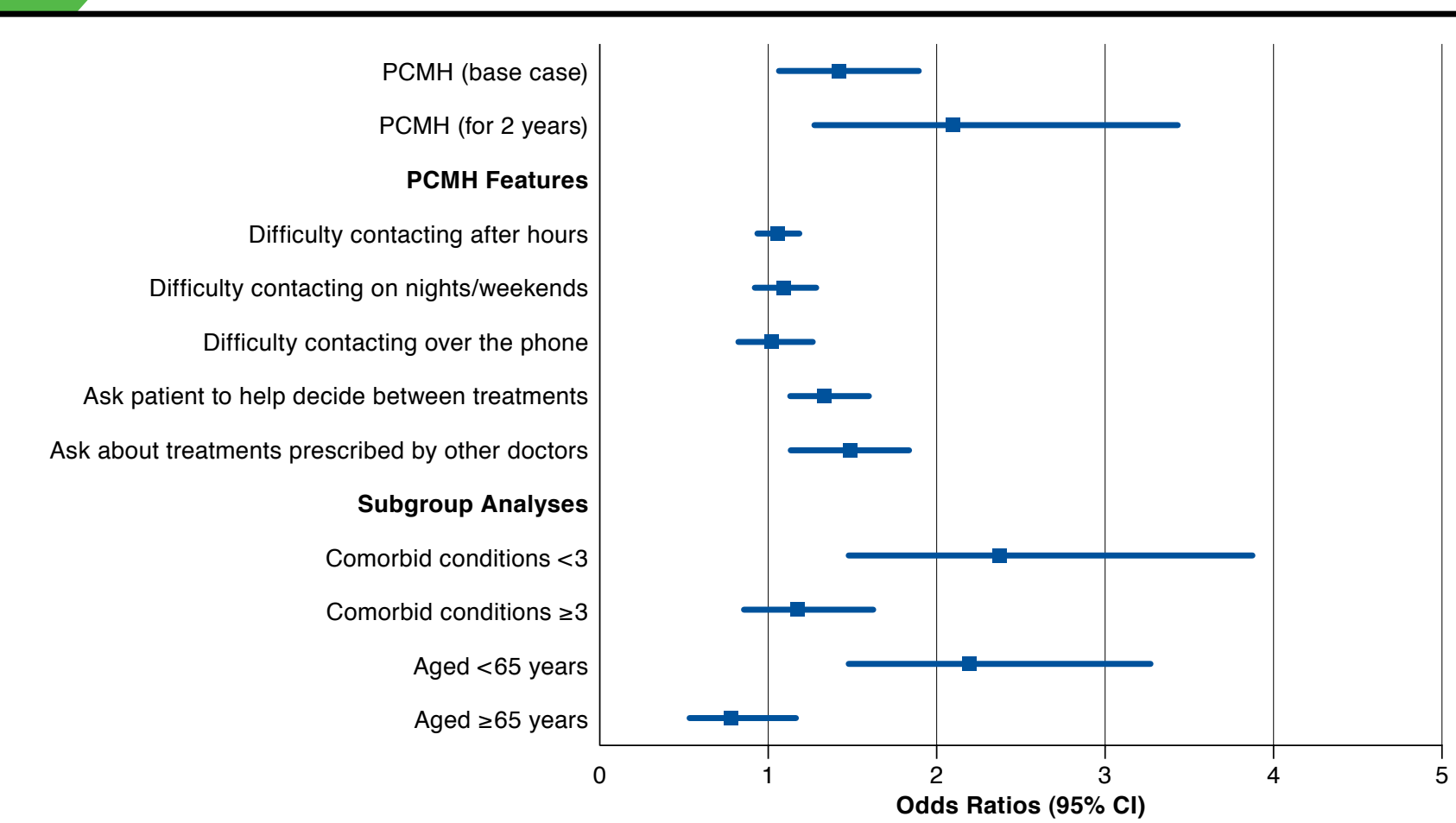

Note: For odds ratios, age, sex, racelethnicity, education, perceived health, years of diabetes, number of comorbidity, depression variables were adjusted. $C I=$ confidence interval; $P C M H=$ patient-centered medical home.

Subgroup analyses suggest that the PCMH model is more effective with patients who have less comorbidities, as well as with younger patients. These findings may be because patients with a single disease or less comorbid conditions usually have less interactions with health care providers so have less opportunity to receive preventive care compared with patients who have multiple chronic conditions. Also, younger patients or patients with less comorbidities tend to be less serious about their condition, which may lead to a greater effect of the PCMH model on the diabetes process measures. ${ }^{31}$ Subgroup analyses of medication adherence suggest different results, although the findings are not statistically significant. The number of comorbid conditions seems to affect medication adherence, with a higher number of comorbid conditions having more of an effect than a lower number of conditions. These findings also suggest that the PCMH model affects various outcomes differently. The process measures of diabetes care are probably more related to interaction with the health care system, while medication adherence is more associated with a person's self-control. Although patients interact more with clinicians, patients with multiple comorbidities may have a hard time managing and adhering to multiple medications. The effect of the PCMH model on medication adherence and other measures needs to be further explored.
From the multivariate analysis, we confirmed several widely known factors associated with adherence. Racial disparities were found in this study. Black and Hispanic patients were less likely to adhere to the ADA-recommended diabetes care process measures. Higher education was associated with better performance of process measures. Patients who stated that they were in good health were associated with better performance, which may be interpreted as health consciousness. Longer duration of diabetes and higher Elixhauser comorbidity scores can be interpreted as severity of diseases, and patients with severe disease conditions were more likely to meet the process measure criteria. Many of these covariates were not statistically significant in the medication adherence analysis. Higher copayment amount was associated with better adherence. These findings may be counterintuitive; however, this variable showed how much money that an individual actually paid in the baseline year rather than the amount that the individual had to pay in the follow-up year. Therefore, this variable may be related to how well a patient filled his or her prescriptions at baseline.

This study provides particular patient perspectives of the PCMH model. Current PCMH evaluation studies are mostly from pilot projects or from PCMHs certified by the NCQA. Although patient-reported PCMH principles may not 


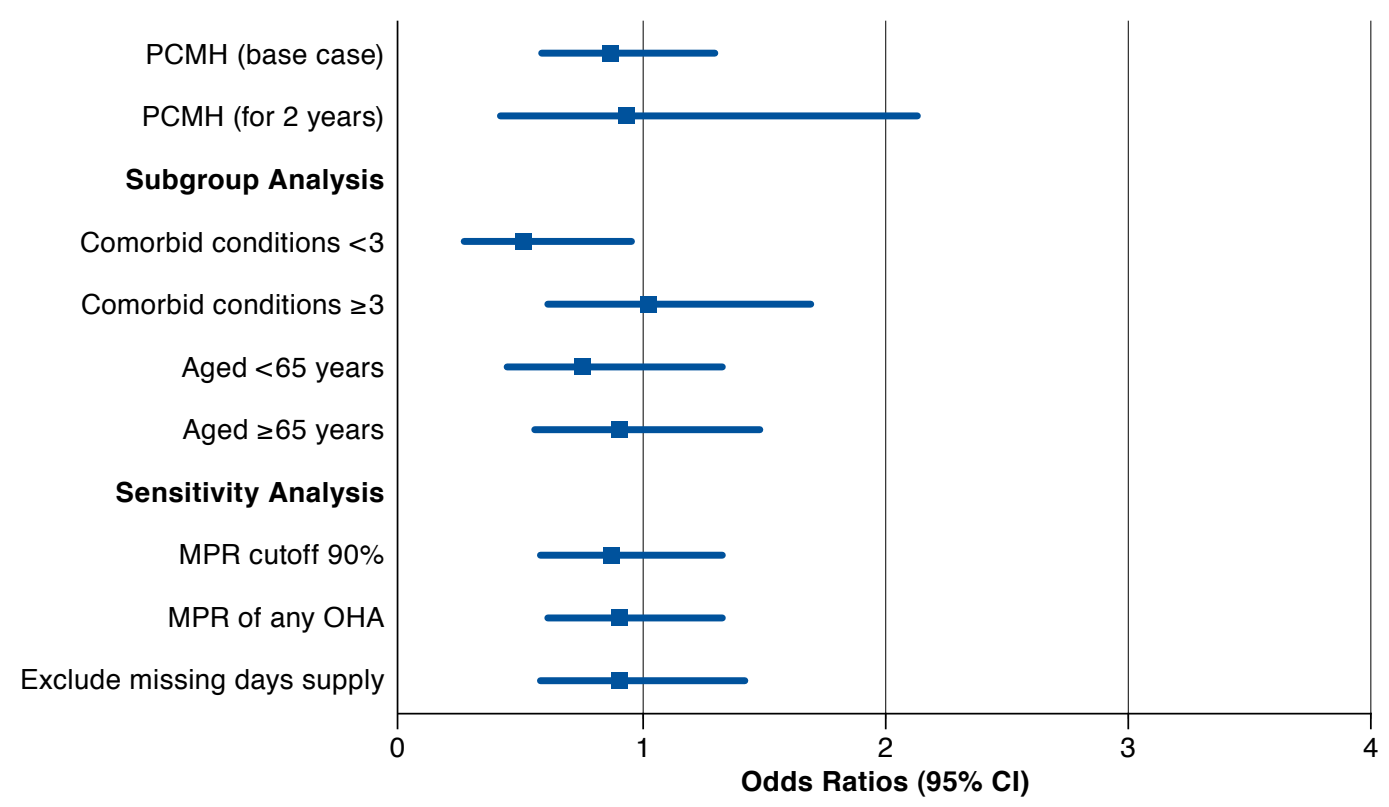

Note: For odds ratios, age, sex, racelethnicity, education, perceived health, years of diabetes, number of comorbidity, depression variables were adjusted. $C I=$ confidence interval; $M P R=$ medication possession ratio; $O H A=$ oral hypoglycemic agent; $P C M H=$ patient-centered medical home.

necessarily be consistent with PCMH certification, patients may receive a higher quality of care that is consistent with PCMH principles before formal recognition. ${ }^{32}$ In addition, self-reported outcomes provide unique patient perspectives on the PCMH model. ${ }^{5}$ The data for each PCMH feature provide the opportunity to further understand the effect of the PCMH model. Several studies that investigated PCMHs using survey data indicated that survey data based on patient reporting can reliably predict outcomes similar to existing evidence. .,21,23-25 $^{-1}$

This study showed that positive responses in shared decision making and enhanced patient engagement questions seem to be highly associated with the process measures of diabetes care. Asking patients to help decide treatment and asking about treatments by other doctors indicated a higher likelihood of improvement in the process measure; however, difficulty in contacting usual care providers was not associated with the process measure outcome. These findings are related to previous findings using the same data source. Stockbridge et al. (2014) reported higher pharmacy expenditures for individuals whose providers asked about medications and treatments prescribed by other doctors. ${ }^{21}$ However, this study also found lower total expenditures for the individuals who had no difficulty in contacting the regular source of care over the telephone during regular business hours. Again, this shows that each PCMH feature may influence various outcomes differently.

\section{Limitations}

This study has several limitations. The definition of PCMHs was based on patient responses. Although this study provides unique patient perspectives on PCMHs, self-reported patient perceptions may not accurately define the PCMH model. Some PCMH principles have not been captured in MEPS (e.g., adoption of electronic medical records, use of evidence-based clinical protocols, and degree of care coordination when transitioning between different levels of care) or may not be familiar to patients, which may lead to bias in the measurement. The number of questionnaires used to define PCMHs in this study are relatively smaller than the questionnaires used to define PCMHs among the pediatric population using the MEPS data. ${ }^{23-25}$

As with other observational studies, this study's findings may be biased because of potential confounding factors. In this study, multivariate analysis was used to control for observed confounding factors; however, unmeasured confounders such as successful provider-patient relationships that are not necessarily characteristics of PCMHs might lead to the study findings. This study suffers from typical nonrespondent bias or recall bias that are present in survey studies. As in other survey studies, this analysis had to rely on available data.

The medication adherence outcome was measured using MPR from the MEPS pharmacy files. Although the MEPS 
prescription drug data are reportedly accurate, ${ }^{33}$ missing values in days supply records and unavailable fill dates are limitations of studying medication adherence using MEPS. To overcome this, MPRs were calculated in different ways, and sensitivity analyses were conducted around the definition of medication adherence. The study population was restricted to patients who initiated OHAs, and insulin users were excluded, since accurate calculation of refill adherence is challenging for insulin users.

Despite these limitations, this study has several strengths. It is one of the few studies examining the PCMH effect on medication adherence in a nationally representative U.S. sample. This study used panel data to investigate the association between PCMHs and process measures of diabetes care, along with medication adherence, to avoid the typical limitations of a cross-sectional survey study. Unique patient perspectives on PCMHs for various outcomes further provided insights regarding implementation of PCMHs for the broader population.

\section{Conclusions}

The PCMH model was associated with improvement in the process measures of diabetes care, potentially driven by enhanced shared decision making and patient engagement. However, the PCMH model was not associated with increased adherence to OHAs. Future studies should further explore the effect of PCMHs on various outcomes to understand these mixed findings and to suggest changes in the health care system to improve patient adherence.

\section{Authors}

JAEJIN AN, BPharm, PhD, Department of Pharmacy Practice and Administration, Western University of Health Sciences College of Pharmacy, Pomona, California.

AUTHOR CORRESPONDENCE: JaeJin An, BPharm, PhD, Department of Pharmacy Practice and Administration, Western University of Health Sciences College of Pharmacy, 309 E. Second St., Pomona, CA 91766. Tel.: 909.469.8778; Fax: 909.469.5428; E-mail: jan@westernu.edu.

\section{DISCLOSURES}

No outside funding supported this study. The author reports no conflicts of interests.

\section{REFERENCES}

1. Peikes D, Zutshi A, Genevro JL, Parchman ML, Meyers DS. Early evaluations of the medical home: building on a promising start. Am J Manag Care. 2012;18(2):105-16.

2. Sia C, Tonniges TF, Osterhus E, Taba S. History of the medical home concept. Pediatrics. 2004;113(5 Suppl):1473-78.
3. Kilo CM, Wasson JH. Practice redesign and the patient-centered medical home: history, promises, and challenges. Health Aff (Millwood). 2010;29(5):773-78.

4. American Academy of Family Physicians, American Academy of Pediatrics, American College of Physicians, American Osteopathic Association. Joint principles of a patient-centered medical home. March 2007. Available at: http://www.aafp.org/dam/AAFP/documents/practice_management/pcmh/ initiatives/PCMHJoint.pdf. Accessed October 1, 2016.

5. Pourat N, Charles SA, Snyder S. Availability of care concordant with patient-centered medical home principles among those with chronic conditions: measuring care outcomes. Med Care. 2016;54(3):262-68.

6. Jackson GL, Powers BJ, Chatterjee R, et al. Improving patient care. The patient centered medical home. A systematic review. Ann Intern Med. 2013;158(3):169-78

7. Hoff T, Weller W, DePuccio M. The patient-centered medical home: a review of recent research. Med Care Res Rev. 2012;69(6):619-44.

8. Kern LM, Edwards A, Kaushal R. The patient-centered medical home, electronic health records, and quality of care. Ann Intern Med. 2014;160(11):741-49.

9. Rosenthal MB, Alidina S, Friedberg MW, et al. Impact of the Cincinnati Aligning Forces for Quality multi-payer patient centered medical home pilot on health care quality, utilization, and costs. Med Care Res Rev. 2016;73(5):532-45.

10. Rosenthal MB, Sinaiko AD, Eastman D, Chapman B, Partridge G. Impact of the Rochester Medical Home Initiative on primary care practices, quality, utilization, and costs. Med Care. 2015;53(11):967-73.

11. Rosenthal MB, Alidina S, Friedberg MW, et al. A difference-in-difference analysis of changes in quality, utilization and cost following the Colorado Multi-Payer Patient-Centered Medical Home Pilot. J Gen Intern Med. 2016;31(3):289-96.

12. Friedberg MW, Rosenthal MB, Werner RM, Volpp KG, Schneider EC. Effects of a medical home and shared savings intervention on quality and utilization of care. JAMA Intern Med. 2015;175(8):1362-68.

13. Friedberg MW, Schneider EC, Rosenthal MB, Volpp KG, Werner RM Association between participation in a multipayer medical home intervention and changes in quality, utilization, and costs of care. JAMA. 2014;311(8):815-25

14. David G, Gunnarsson C, Saynisch PA, Chawla R, Nigam S. Do patientcentered medical homes reduce emergency department visits? Health Serv Res. 2015;50(2):418-39.

15. Dale SB, Ghosh A, Peikes DN, et al. Two-year costs and quality in the Comprehensive Primary Care Initiative. N Engl J Med. 2016;374(24):2345-56.

16. Cole ES, Campbell C, Diana ML, Webber L, Culbertson R. Patientcentered medical homes in Louisiana had minimal impact on Medicaid population's use of acute care and costs. Health Aff (Millwood). 2015;34(1):87-94.

17. Beadles CA, Farley JF, Ellis AR, et al. Do medical homes increase medication adherence for persons with multiple chronic conditions? Med Care. 2015;53(2):168-76.

18. Domino ME, Humble C, Lawrence WW Jr, Wegner S. Enhancing the medical homes model for children with asthma. Med Care. 2009;47(11):1113-20.

19. Beadles CA, Hassmiller Lich K, Viera AJ, Greene SB, Brookhart MA, Weinberger M. Patient-centered medical homes and oral anticoagulation therapy initiation. Med Care Res Rev. 2014;71(2):174-91.

20. McGinley EL, Gabbay RA. The impact of new payment models on quality of diabetes care and outcomes. Curr Diab Rep. 2016;16(6):51.

21. Stockbridge EL, Philpot LM, Pagán JA. Patient-centered medical home features and expenditures by medicare beneficiaries. Am J Manag Care. 2014:20(5):379-85. 
22. Stagnitti, MN. Trends in utilization and expenditures of prescribed drugs treating diabetes, hypertension, and high cholesterol for persons under age 40 in the U.S. civilian noninstitutionalized population, 2000 and 2010. Statistical Brief \#407. June 2013. Agency for Healthcare Research and Quality, Rockville, MD. Available at: http://meps.ahrq.gov/mepsweb/data_ files/publications/st407/stat407.shtml. Accessed October 1, 2016.

23. Romaire MA, Bell JF, Grossman DC. Medical home access and health care use and expenditures among children with special health care needs. Arch Pediatr Adolesc Med. 2012;166(4):323-30.

24. Romaire MA, Bell JF, Grossman DC. Health care use and expenditures associated with access to the medical home for children and youth. Med Care. 2012;50(3):262-69.

25. Han B, Yu H, Friedberg MW. Evaluating the impact of parent-reported medical home status on children's health care utilization, expenditures, and quality: a difference-in-differences analysis with causal inference methods. Health Serv Res. June 3, 2016. Epub ahead of print.

26. American Diabetes Association. Strategies for improving care. Sec. 1. In: Standards of medical care in diabetes 2016. Diabetes Care. 2016;39 (Suppl 1):S6-S12
27. Peterson AM, Nau DP, Cramer JA, Benner J, Gwadry-Sridhar F, Nichol M. A checklist for medication compliance and persistence studies using retrospective databases. Value Health. 2007;10(1):3-12.

28. Elixhauser A, Steiner C, Harris DR, Coffey RM. Comorbidity measures for use with administrative data. Med Care. 1998;36(1):8-27.

29. Thompson NR, Fan Y, Dalton JE, et al. A new Elixhauser-based comorbidity summary measure to predict in-hospital mortality. Med Care. 2015;53(4):374-79.

30. Goodman RA, Posner SF, Huang ES, Parekh AK, Koh HK. Defining and measuring chronic conditions: imperatives for research, policy, program, and practice. Prev Chronic Dis. 2013;10:E66. Published online April 25, 2013 Available at: https://www.ncbi.nlm.nih.gov/pmc/articles/PMC3652713/. Accessed October 1, 2016.

31. Juth V, Silver RC, Sender L. The shared experience of adolescent and young adult cancer patients and their caregivers. Psychooncology. 2015;24(12):1746-53

32. Pourat N, Hadler M. Ready for ACA? How community health centers are preparing for health care reform. Policy Brief UCLA Cent Health Policy Res. 2014;(PB2014-4):1-6, appendix.

33. Hill SC, Zuvekas SH, Zodet MW. Implications of the accuracy of MEPS prescription drug data for health services research. Inquiry. 2011;48(3):242-59. 


\section{APPENDIX A Univariate and Multivariate Results of Process Measures of Diabetes Care}

\begin{tabular}{|c|c|c|}
\hline & \multicolumn{2}{|c|}{ OR $(95 \% \mathrm{CI})$} \\
\hline & Univariate & Multivariate \\
\hline \multicolumn{3}{|c|}{ Outcome: overall process measures of diabetes care ${ }^{a}$} \\
\hline Patient-centered medical homes (yes vs. no) & $1.39 \quad(0.94-2.07)$ & $1.42 \quad(1.06-1.91)$ \\
\hline \multicolumn{3}{|l|}{ Age category } \\
\hline Aged $\geq 65$ years (vs. aged $18-44$ years) & $2.21 \quad(1.45-3.37)$ & $1.08 \quad(0.77-1.50)$ \\
\hline Aged 45-64 (vs. aged 18-44 years) & $1.94 \quad(1.23-3.04)$ & $1.08 \quad(0.76-1.53)$ \\
\hline Male (vs. female) & $0.97 \quad(0.76-1.24)$ & $0.91 \quad(0.73-1.13)$ \\
\hline \multicolumn{3}{|l|}{ Race/ethnicity } \\
\hline Hispanic (vs. white) & $0.51 \quad(0.39-0.65)$ & $0.59 \quad(0.46-0.77)$ \\
\hline Black (vs. white) & $0.53 \quad(0.41-0.68)$ & $0.58 \quad(0.46-0.73)$ \\
\hline Asian/others (vs. white) & $0.58 \quad(0.36-0.94)$ & $0.61 \quad(0.40-0.95)$ \\
\hline \multicolumn{3}{|l|}{ Education } \\
\hline College or more (vs. lower than high school) & $0.98 \quad(0.73-1.33)$ & $1.25 \quad(0.96-1.63)$ \\
\hline High school (vs. lower than high school) & $1.79 \quad(1.35-2.37)$ & $1.77 \quad(1.38-2.28)$ \\
\hline \multicolumn{3}{|l|}{ Perceived health } \\
\hline Excellent/very good (vs. fair/poor) & $1.18 \quad(0.78-1.79)$ & $1.37 \quad(0.97-1.92)$ \\
\hline Good (vs. fair/poor) & $1.55 \quad(1.21-1.98)$ & $1.50 \quad(1.19-1.89)$ \\
\hline \multicolumn{3}{|l|}{ Years of diabetes } \\
\hline$>10$ years (vs. $<5$ years) & $2.36 \quad(1.72-3.25)$ & $2.64 \quad(1.99-3.50)$ \\
\hline $5-10$ years (vs. $<5$ years) & $1.38 \quad(0.96-1.98)$ & $1.48 \quad(1.05-2.09)$ \\
\hline Not available (vs. $<5$ years) & $0.85 \quad(0.33-2.19)$ & $0.65 \quad(0.26-1.62)$ \\
\hline Elixhauser comorbidity score & $1.03 \quad(1.01-1.05)$ & $1.02 \quad(1.00-1.04)$ \\
\hline Depression (yes vs. no) & $0.78 \quad(0.56-1.09)$ & $0.88 \quad(0.68-1.15)$ \\
\hline \multicolumn{3}{|l|}{ Outcome: each process measure of diabetes care } \\
\hline Alc test & $1.02 \quad(0.73-1.43)$ & $1.04 \quad(0.78-1.39)$ \\
\hline Cholesterol test & $1.18 \quad(0.75-1.84)$ & $0.96 \quad(0.64-1.42)$ \\
\hline Diabetes foot examination & $1.39 \quad(0.94-2.06)$ & $1.21 \quad(0.84-1.75)$ \\
\hline Dilated eye examination & $1.51 \quad(1.05-2.18)$ & $1.49 \quad(1.08-2.05)$ \\
\hline Flu vaccination & $1.13 \quad(0.77-1.65)$ & $1.14 \quad(0.80-1.61)$ \\
\hline
\end{tabular}




\section{APPENDIX B Univariate and Multivariate Results of Adherence to OHAs}

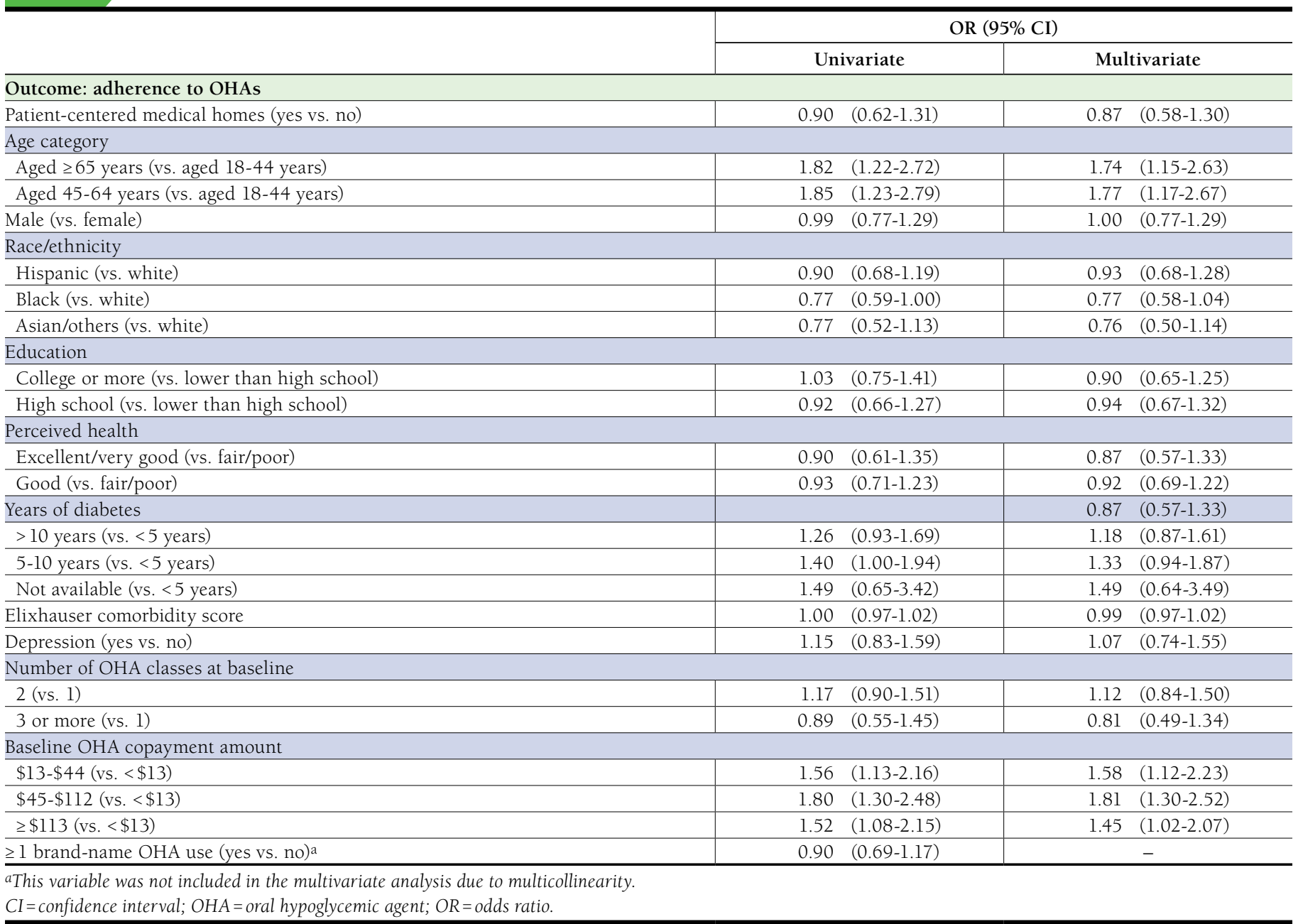

